\title{
Efeito do manejo de aleitamento nos níveis de cortisol no metabolismo e na produção de leite de vacas holandesas
}

\author{
Fernanda Alves de Paiva1, João Alberto Negrão², Arlindo Saran Netto³, Marco Aurélio de \\ Felicio Porcionato ${ }^{4}$, César Gonçalves de Lima ${ }^{5}$
}

\begin{abstract}
1 Doutoranda FZEA/USP, Av. Duque de Caxias Norte, 225, Caixa Postal 23, CEP: 13630-000 - Pirassununga, SP.
2 FZEA/USP, Av. Duque de Caxias Norte, 225, Caixa Postal 23, CEP: 13630-000 - Pirassununga, SP.

3 Doutorando FZEA/USP, Av. Duque de Caxias Norte, 225, Caixa Postal 23, CEP: 13630-000 - Pirassununga, SP.

${ }^{4}$ Doutorando FCAVIUNESP, Av. Duque de Caxias Norte, 225, Caixa Postal 23, CEP: 13630-000 - Pirassununga, SP.

${ }^{5}$ Estatístico, FZEA/USP, Av. Duque de Caxias Norte, 225, Caixa Postal 23, CEP: 13630-000 - Pirassununga, SP.
\end{abstract}

RESUMO - Avaliou-se a influência do manejo de aleitamento nos níveis de cortisol, no metabolismo e na produção de leite de vacas leiteiras. Utilizaram-se 18 vacas holandesas e seus bezerros alocados em três tratamentos: T1: as vacas foram separadas dos bezerros 6 horas após o parto, sendo mantidas com suas crias 60 minutos por dia nos três primeiros dias; T2 as vacas foram separadas dos bezerros 6 horas após o parto; T3: as vacas foram mantidas com seus bezerros durante os três primeiros dias de lactação, exceto no momento das ordenhas. Foram coletadas amostras de sangue 168 horas antes do parto, no dia do parto (0 hora) e 24, 48, 72 e 96 horas após o parto para a determinação de glicose, triglicerídeos, proteína total e cortisol. A produção leiteira foi mensurada duas vezes ao dia e a porcentagem de leite residual foi estimada nas duas ordenhas posteriores à desmama. As concentrações de cortisol foram maiores ao parto, contudo, não houve diferença significativa entre os tratamentos. Durante o período experimental, as vacas separadas dos bezerros apresentaram maior produção de leite, porém, após este período, não houve diferença entre os tratamentos. Após a desmama, a porcentagem de leite residual foi maior em T1 e T3, demonstrando que o uso de ordenha mecânica exclusiva após um período de amamentação prejudicou a ejeção do leite, mesmo em vacas especializadas. Os níveis de glicose foram menores em T2 às 72 horas e as concentrações de triglicerídeos foram menores em T3 às 72 e 96 horas. Os manejos estudados não influenciaram os níveis de cortisol e o metabolismo das vacas e nem prejudicaram a produção leiteira após o período colostral.

Palavras-chave: amamentação, desmama, estresse, leite residual, ordenha mecanizada

\section{Effects of different milking management on metabolism, cortisol levels, and milk yield of Holstein cows}

\begin{abstract}
The effects of different milking management on metabolism, cortisol levels, and milk yield of Holstein cows were investigated in this trial. Eighteen Holstein cows and calves were randomly assigned to one of the following three treatments: T1) cows were separated from their calves $6 \mathrm{~h}$ after parturition but reunited with them for two daily periods of 30 minutes each; T2) cows were separated from their calves $6 \mathrm{~h}$ after parturition; or T3) cows were maintained with their calves during the first three days of lactation except at milking times. Blood samples were collected $168 \mathrm{~h}$ before parturition, at parturition $(0 \mathrm{~h})$ and at 24, 48, 72 and $96 \mathrm{~h}$ after parturition and analyzed for glucose, triglycerides, total protein, and cortisol. Milk yield was measured twice a day and the percentage of residual milk was estimated based on the first two milkings after weaning. Although plasma cortisol levels were greater at parturition $(0 \mathrm{~h})$ compared to the remaining sampling points, no significant differences were observed among treatments. Cows on T2 yielded more milk than those on T1 and T3 during the first four days of lactation with no difference in milk production thereafter. After weaning, the percentage of residual milk was higher on T1 and T3 showing that the exclusive use of milking machine after a nursing period appeared to negatively affect milk ejection. Concentration of plasma glucose was lower at $72 \mathrm{~h}$ on T2 while that of triglycerides was lower at 72 and $96 \mathrm{~h}$ on T3. The studied managements did not influence the cortisol levels and metabolism of cows and did not prejudice the milk production after the colostral period.
\end{abstract}

Key Words: nursing, milking machine, residual milk, stress, weaning

\section{Introdução}

As vacas especializadas na produção de leite podem ser submetidas à ordenha sem a presença de seus bezerros (Negrão \& Marnet, 2002). Normalmente, em fazendas comerciais, os bezerros são separados de suas mães logo 
após o nascimento, recebendo colostro e/ou leite artificialmente. Contudo, em algumas fazendas, permite-se que o bezerro permaneça com sua mãe por um ou até três dias de vida, retirando-se a vaca apenas no momento da ordenha para esgotamento do úbere como forma de garantir aquisição adequada de imunidade passiva pelo bezerro (Nocek et al., 1984; Fallow et al., 1989).

Entretanto, este tipo de manejo na fase colostral pode comprometer a produção de leite nas ordenhas subseqüentes à desmama (Marnet \& Negrão, 2000). Isso ocorre porque os ruminantes reconhecem seus filhotes rapidamente após o parto e qualquer separação após 12 horas de contato pode ser considerada estressante (Poidron \& Signoret, 1976; Poidron et al., 1980). Neste caso, em vez da sensação de conforto normalmente promovida pela ordenha, em que há liberação de ocitocina e ejeção do leite via reflexo neuroendócrino (Gorewit et al., 1983; Marnet et al., 1998), pode haver inibição do reflexo de ejeção de leite, mesmo em vacas especializadas (Bruckmaier et al., 1993; Tancin et al., 1995). Este tipo de estresse inibe a síntese e liberação de ocitocina pela neurohipófise, de modo que a administração de doses fisiológicas de ocitocina durante a ordenha pode restabelecer a ejeção do leite (Bruckmaier \& Blum, 1996).

Logo após o parto, as vacas leiteiras especializadas também entram em um período de balanço energético negativo, proporcional à sua produção leiteira (Castilho et al., 2005). Neste caso, animais mais produtivos apresentam alterações metabólicas que caracterizam um processo de lipólise importante (Drackley, 2002), promovendo conseqüentemente, aumento nos níveis plasmáticos de betahidroxibutirato e ácidos graxos não-esterificados e diminuição nos níveis plasmáticos de glicose (Dann et al., 2005; Odensten et al., 2005).

O objetivo neste trabalho foi avaliar o efeito de diferentes manejos de aleitamento nos níveis de cortisol, no metabolismo e na produção de leite de vacas especializadas.

\section{Material e Métodos}

$\mathrm{O}$ experimento foi desenvolvido entre maio e agosto de 2001, na Faculdade de Zootecnia e Engenharia de Alimentos da Universidade de São Paulo, no Campus Administrativo de Pirassununga (latitude 21 o 59' S, longitude $47^{\circ} 26^{\prime} \mathrm{W}$ e altitude de $634 \mathrm{~m}$ ). Nesta região, o clima é subtropical do tipo Cwa de Köppen (Oliveira \& Prado, 1984).

Foram utilizadas 18 vacas holandesas, com seus respectivos bezerros, em fase final de gestação e início de lactação. Os tratamentos consistiram de três diferentes manejos de ordenha: no tratamento 1 (T1), as vacas foram separadas dos bezerros 6 horas após o parto, sendo mantidas com suas crias por dois períodos de 30 minutos, durante os três primeiros dias pós-parto. Após a amamentação, as vacas foram submetidas à ordenha mecanizada; no tratamento 2 (T2), foram separadas dos bezerros 6 horas após o nascimento e, durante os três primeiros dias pós-parto, foram submetidas apenas à ordenha mecanizada exclusiva; no tratamento 3 (T3), foram mantidas durante os três primeiros dias pós-parto com os bezerros, em piquete isolado dos demais animais e, duas vezes ao dia, foram retiradas do piquete para serem ordenhadas. A desmama nos tratamentos 1 e 3 foi realizada 72 horas após o parto.

Todas as vacas experimentais pariram naturalmente, sem nenhum tipo de interferência humana, e foram submetidas a duas ordenhas diárias. A produção leiteira foi quantificada diariamente durante os primeiros 30 dias de lactação. Para o cálculo da porcentagem de leite residual, as vacas foram ordenhadas normalmente no $4 \underline{0}$ dia de lactação e receberam, ao término de cada ordenha, 50 UI de ocitocina, via intramuscular. Sete minutos após a administração de ocitocina, as vacas foram novamente ordenhadas para a recuperação do leite residual. A quantidade de leite residual foi calculada utilizando-se a seguinte fórmula: leite residual $(\%)=(L R * 100) /(L L+L R)$, em que $L R=$ leite residual obtido após a aplicação de ocitocina e a nova ordenha; LL = leite liberado durante a ordenha normal.

As coletas de sangue foram realizadas na veia mamária sete dias antes da data prevista do parto (-168 horas), no parto (0 hora), 24, 48, 72 e 96 horas após o parto. O sangue foi centrifugado por 15 minutos $\left(1.500 \mathrm{~g} \mathrm{a} 4^{\circ} \mathrm{C}\right)$ e o plasma obtido foi conservado a $-20^{\circ} \mathrm{C}$ para posterior análise. As concentrações de glicose, proteína total e triglicerídeos foram determinadas utilizando-se kits enzimáticos comerciais (Laborlab) e a concentração de cortisol foi determinada por meio de um kit imunoenzimático comercial (Diagnostic Sistems Laboratories, Inc).

As rações fornecidas nas fases pré e pós-parto foram compostas de concentrado (milho, casca de soja, soja extrusada, fosfato bicálcico e calcário) e silagem de milho. As vacas tiveram livre acesso à sombra, à água e ao suplemento mineral e vitamínico. Durante o período seco e no período experimental, as vacas receberam as mesmas dietas, sempre de acordo com a fase reprodutiva e de produção, segundo recomendações do NRC (2001).

O delineamento experimental utilizado foi em blocos ao acaso, com seis repetições, em que os blocos foram determinados em função da ordem de parto das vacas, avaliadas individualmente em seis ocasiões $(-168,0,24,48,72$ e 96 horas). No modelo de análise com medidas repetidas, foram incluídos os efeitos de bloco,tratamento, tempo e interação 
tratamento $\times$ tempo, sendo escolhida uma estrutura de covariâncias adequada a cada uma das respostas (cortisol, glicose, proteína total e triglicerídeos). Para executar as análises, utilizou-se o Proc Mixed do SAS System for Windows versão 8.12.(2000) e o teste ta 10\% de significância para comparação das médias dos tratamentos.

\section{Resultados e Discussão}

A produção leiteira dos primeiros 30 dias de lactação não foi influenciada pelos diferentes tratamentos (Figura 1), exceto no período experimental (Tabela 1). Neste período, as vacas separadas dos bezerros logo após o parto (T2) tiveram maior produção de leite e menor porcentagem de leite residual após a ordenha quando comparadas às dos demais tratamentos (T1 e T3).

A maior produção de leite quantificada na ordenha das vacas cujos bezerros foram desmamados logo após o parto (T2) resultou da ausência de amamentação, sendo todo leite recuperado nas ordenhas (Sandoval-Castro et al., 1999). Já a maior porcentagem de leite residual obtida em vacas que tiveram contato com seus bezerros (T1 e T3) provavelmente foi ocasionada por menor liberação de ocitocina e, conseqüentemente, menor ejeção do leite (Bruckmaier et al., 1993; Tancin et al., 1995; Negrão, 1996). Outros autores, que também trabalharam com vacas, observaram que as primeiras ordenhas executadas após a desmama foram acompanhadas de deficiência na liberação de ocitocina e retenção de leite residual (Negrão \& Marnet, 2002)

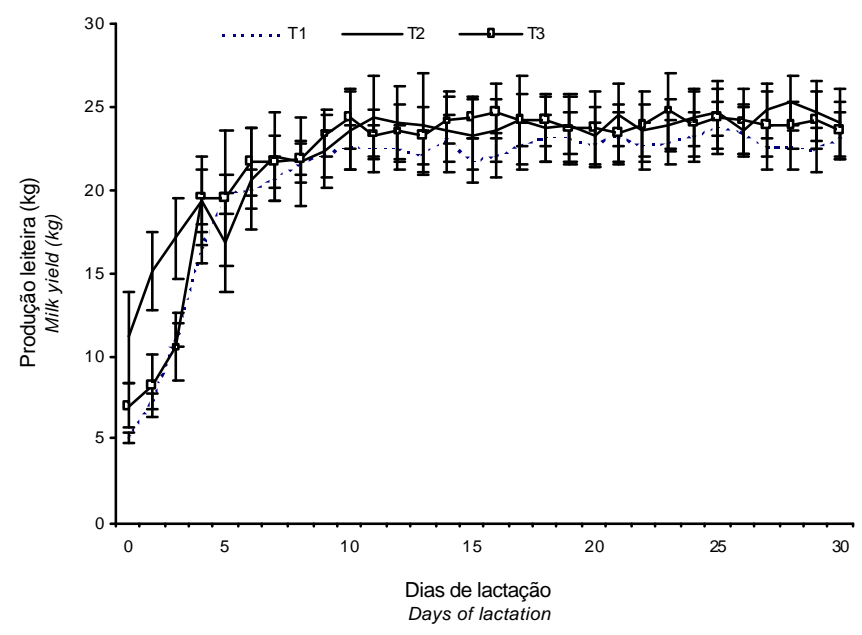

Figura 1 - Produção leiteira (média \pm erro padrão ${ }^{1}$ ) nos 30 primeiros dias de lactação de vacas holandesas nos diferentes tratamentos.

Figure 1 - Milk yield (mean \pm standard error ${ }^{1}$ ) measured in the first 30 days of lactation of Holstein cows submitted to different milking management.
Tabela 1 - Produção leiteira (média \pm erro-padrão $^{1}$ ) nos quatro primeiros dias de lactação de vacas holandesas nos diferentes tratamentos

Table 1 - Milk yield (mean \pm standard error ${ }^{1}$ ) in the first four days of lactation of Holstein cows submitted to different milking management

\begin{tabular}{lcrrr}
\hline \multirow{2}{*}{$\begin{array}{l}\text { Parâmetro } \\
\text { Item }\end{array}$} & \multirow{2}{*}{$\begin{array}{c}\text { Dia } \\
\text { Day }\end{array}$} & \multicolumn{3}{c}{$\begin{array}{c}\text { Tratamento } \\
\text { Treatment }\end{array}$} \\
\cline { 2 - 5 } & & \multicolumn{1}{c}{$\mathrm{T} 1$} & \multicolumn{1}{c}{$\mathrm{T} 2$} & \multicolumn{1}{c}{$\mathrm{T} 3$} \\
\hline PL (kg) & 1 & $5,13^{\mathrm{b}} \pm 1,18$ & $11,12^{\mathrm{a}} \pm 2,69$ & $6,98^{\mathrm{b}} \pm 1,33$ \\
& 2 & $7,30^{\mathrm{b}} \pm 1,62$ & $15,10^{\mathrm{a}} \pm 2,41$ & $8,20^{\mathrm{b}} \pm 1,91$ \\
& 3 & $11,30^{\mathrm{b}} \pm 1,48$ & $17,10^{\mathrm{a}} \pm 2,38$ & $10,53^{\mathrm{b}} \pm 2,05$ \\
PL (kg) & 4 & $10,90^{\mathrm{b}} \pm 3,34$ & $17,20^{\mathrm{a}} \pm 2,65$ & $13,03^{\mathrm{b}} \pm 1,68$ \\
LR (kg) & & $5,60^{\mathrm{a}} \pm 1,99$ & $2,07^{\mathrm{b}} \pm 0,75$ & $6,53^{\mathrm{a}} \pm 1,12$ \\
LRP $(\%)^{4}$ & & $36,18^{\mathrm{a}} \pm 9,08$ & $9,61^{\mathrm{b}} \pm 2,58$ & $35,40^{\mathrm{a}} \pm 7,48$ \\
\hline
\end{tabular}

${ }^{1}$ Médias seguidas pela mesma letra na linha não diferem pelo teste $(P>0,10)$.

2 PL: produção leiteira (milk yield).

3 LR: leite residual (residual milk).

4 LRP: porcentagem de leite residual (percentage of residual milk).

${ }^{1}$ Means followed by the same letter in a row do not differ $(P>0.10)$ by t test.

Embora as vacas estudadas sejam da raça Holandesa (considerada especializada na produção de leite) e não de pendam dos bezerros para a adequada liberação de ocitocina e a ejeção de leite (Macuhová et al., 2002), a ordenha mecanizada exclusiva provocou, aparentemente, menor liberação de ocitocina quando comparada ao estímulo promovido anteriormente pelos bezerros, que aumentou a porcentagem de leite residual nas vacas dos grupos T1 e T3.

Este tipo de inibição da ejeção foi observado em bovinos (Tancin et al., 1995) e ovinos (Marnet \& Negrão, 2000) mantidos com seus filhotes. Entretanto, a ejeção do leite das vacas do T1 e T3 aumentou gradualmente e, após alguns dias, foi semelhante à produção das vacas do T2, que foram separadas dos bezerros logo após o parto. Conseqüentemente, após o período experimental, não houve diferença entre a produção leiteira dos diferentes grupos.

As concentrações de cortisol foram influenciadas significativamente apenas pelo período de coleta de sangue - as maiores concentrações foram observadas no parto (Figura 2). Ao mesmo tempo, os níveis de cortisol apresentaram perfil semelhante entre os tratamentos ao longo das coletas de sangue. Os níveis de cortisol observados neste trabalho apresentaram, em final de gestação e início de lactação, valores e variabilidade semelhantes aos descritos por outros autores (Hydbring et al., 1999).

O aumento nos níveis de cortisol no parto pode ser considerado normal, visto que o parto é um evento natural que envolve estresse e dor para as mães (Hydbring et al., 1999). Outros autores também observaram aumento nas concentrações de cortisol em vacas no momento do parto (Hudson et al., 1975; Aurich et al., 1993). Essa elevação também pode ser conseqüência da maior necessidade de 


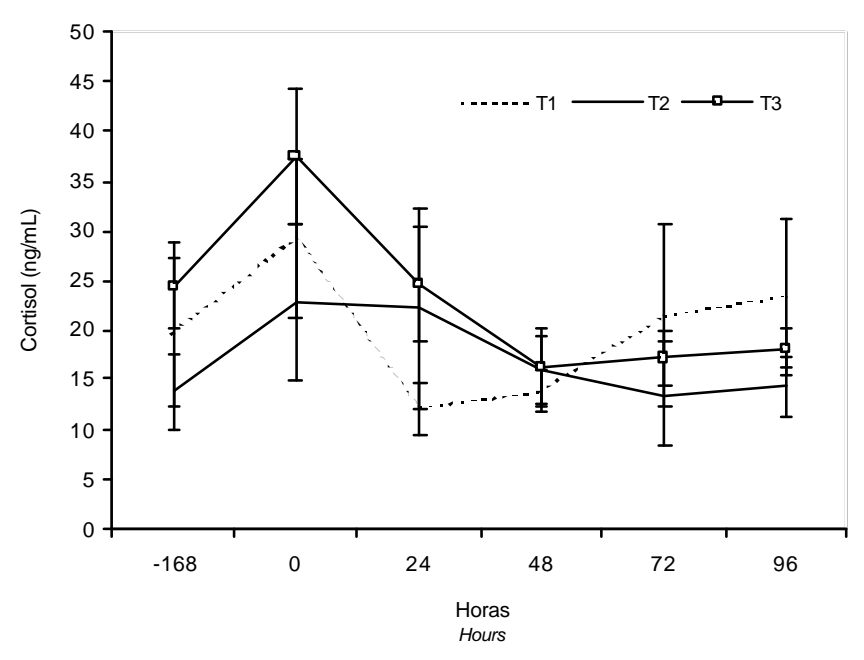

Figura 2 - Concentrações de cortisol (média \pm erro-padrão¹) no plasma de vacas holandesas nos diferentes tratamentos.

Figure 2 - Concentration of plasma cortisol (mean \pm standard error) of Holstein cows submitted to different milking management. glicocorticóides para acelerar o desenvolvimento mamário e iniciar a lactação (Hitts, 1985). Como observado neste trabalho, Hydbring et al. (1999) também notaram que, 24 horas após o parto, os níveis de cortisol retornaram aos valores registrados antes do parto.

Após a desmama, não foi constatado aumento significativo nas concentrações de cortisol neste estudo, o que diverge dos resultados descritos por outros autores (Lidfords, 1996; Tancin et al., 1995) que trabalharam em condições experimentais semelhantes. Contudo, certos grupos genéticos respondem com maior intensidade a de terminados estímulos estressantes (Blecha et al., 1984; Zavy et al., 1992). Mesmo quando a separação ocorreu de 4 a 6 meses pós-parto, período em que o bezerro é pouco dependente da mãe, foi constatado aumento significativo do cortisol após a desmama (Lefcourt \& Elsasser, 1995).

Os níveis plasmáticos de proteína total não foram influenciados pelos tratamentos nem pelos períodos de

Tabela 2 - Concentrações plasmáticas (média \pm erro-padrão ${ }^{1}$ ) de glicose, proteína total e triglicerídeos de vacas holandesas nos diferentes tratamentos

Table 2 - Concentrations of plasma glucose, total protein and triglycerides (mean \pm standard error) of Holstein cows submitted to different milking management

\begin{tabular}{|c|c|c|c|c|}
\hline $\begin{array}{l}\text { Período de coleta } \\
\text { Collection period }\end{array}$ & $\begin{array}{c}\text { Tratamento } \\
\text { Treatment }\end{array}$ & $\begin{array}{c}\text { Glicose }(\mathrm{mg} / \mathrm{dL}) \\
\text { Glucose }\end{array}$ & $\begin{array}{c}\text { Proteína total }(\mathrm{g} / \mathrm{dL}) \\
\text { Total protein }\end{array}$ & $\begin{array}{c}\text { Triglicerídeos }(\mathrm{mg} / \mathrm{dL}) \\
\text { Triglycerides }\end{array}$ \\
\hline-168 & $\begin{array}{l}1 \\
2 \\
3\end{array}$ & $\begin{array}{l}82,75 \pm 4,77 \\
79,77 \pm 1,46 \\
89,03 \pm 1,57\end{array}$ & $\begin{array}{c}6,63 \pm 0,54 \\
6,32 \pm 0,47 \\
6,73 \pm 0,27\end{array}$ & $\begin{array}{r}104,10 \pm 10,40 \\
108,75 \pm 22,43 \\
101,09 \pm 4,17\end{array}$ \\
\hline $0 \mathrm{~h}$ & $\begin{array}{l}1 \\
2 \\
3\end{array}$ & $\begin{array}{l}102,03 \pm 5,37 \\
107,89 \pm 7,99 \\
108,50 \pm 5,10\end{array}$ & $\begin{array}{l}6,02 \pm 0,33 \\
6,65 \pm 0,80 \\
6,94 \pm 0,17\end{array}$ & $\begin{array}{l}123,79 \pm 16,36 \\
114,90 \pm 18,35 \\
110,88 \pm 16,79\end{array}$ \\
\hline $48 \mathrm{~h}$ & $\begin{array}{l}1 \\
2 \\
3\end{array}$ & $\begin{array}{l}78,45 \pm 5,24 \\
70,18 \pm 4,42 \\
76,08 \pm 6,02\end{array}$ & $\begin{array}{l}6,11 \pm 0,63 \\
6,96 \pm 0,85 \\
6,89 \pm 0,38\end{array}$ & $\begin{array}{l}113,43 \pm 11,93 \\
134,03 \pm 13,25 \\
108,27 \pm 13,71\end{array}$ \\
\hline $72 \mathrm{~h}$ & $\begin{array}{l}1 \\
2 \\
3\end{array}$ & $\begin{array}{r}82,54^{\mathrm{a}} \pm 14,25 \\
49,92^{\mathrm{b}} \pm 1,34 \\
68,70^{\mathrm{a}} \pm 10,43\end{array}$ & $\begin{array}{l}6,23 \pm 0,75 \\
6,14 \pm 0,56 \\
6,89 \pm 0,51\end{array}$ & $\begin{array}{r}133,27^{\mathrm{a}} \pm 9,46 \\
152,79^{\mathrm{a}} \pm 10,22 \\
100,72^{\mathrm{b}} \pm 7,65\end{array}$ \\
\hline
\end{tabular}

${ }_{1}^{1}$ Médias seguidas por letras diferentes na coluna diferem $(P<0,10)$ pelo teste $t$.

${ }^{1}$ Means followed by different letters in a column differ $(P<0.10)$ by $t$ test.

coleta, porém, os níveis de triglicerídeos e de glicose foram influenciados pela interação tempo de colheita $\times$ tratamento (Tabela 2). Nas coletas das 72 e 96 horas após o parto, os níveis de triglicerídeos das vacas que permaneceram com seus bezerros (T3) foram inferiores aos observados nas demais. Os níveis de glicose das vacas mantidas separadas dos bezerros (T2) foram menores que o daquelas mantidas por algum tempo com seus bezerros (T1 e T3).
As concentrações de glicose e proteína total encontradas neste estudo foram semelhantes às observadas por Castillo et al. (2005), em estudo com vacas holandesas em final de gestação e início de lactação. Entretanto, as concentrações de triglicerídeos observadas neste trabalho foram superiores às encontradas por esses autores $(39,71$ a $51,79 \mathrm{mg} / \mathrm{dL}$ ), provavelmente porque, no trabalho citado, as vacas receberam dieta com nível de energia superior ao 
recomendado pelo NRC no período pré-parto, na tentativa de amenizar o balanço energético negativo ocasionado pela diminuição na ingestão de MS antes e logo após o parto. Segundo Drackley (2002), a menor ingestão de MS neste período causa mudanças no metabolismo lipídico, podendo ocasionar aumento na concentração de triglicerídeos no sangue, o que possivelmente ocorreu neste estudo, visto que as recomendações do NRC (2001) foram obedecidas.

\section{Conclusões}

Os manejos estudados não influenciaram os níveis de cortisol e o metabolismo das vacas e nem prejudicaram a produção leiteira após o período colostral.

\section{Literatura Citada}

AURICH, J.; DOBRINSKI, I.; HOPPEN, H. et al. Stimulation of release beta-endorphin and oxytocin by prostaglandin $\mathrm{F} 2$ alpha in cattle at parturition. Journal of Reproduction and Fertility, v.89, n.2, p.605-612, 1993.

BLECHA, F.; BOYLES, S.L.; RILEY, J.G. Shipping suppresses lymphocytes blastogenic responses in Angus and Brahman $\mathrm{x}$ Angus feeder calves. Journal of Animal Science, v.59, n.3, p.576-581, 1984

BRUCKMAIER, R.M.; SCHAMS, K.; BLUM, J.W. Milk removal in familiar and unfamiliar surroundings: concentrations of oxytocin; prolactin; cortisol and $\beta$-endorphin. Journal of Dairy Research, v.60, n.4, p.449-456, 1993.

BRUCKMAIER, R.M.; BLUM, J.W. Normal and disturbed milk removal in dairy cows. In: SYMPOSIUM ON MILK SYNTHESIS; SECRETION AND REMOVAL IN RUMINANTS, 1996, Berna. Proceedings... Berna: School of Veterinary Medicine, 1996. p.37-42.

CASTILLO, C.; HERNANDEZ, J.; BRAVO, A. et al. Oxidative status during late pregnancy and early lactation in dairy cows. The Veterinary Journal, v.169, n.2, p.286-292, 2005.

DANN, H.M.; MORIN, D.E.; BOLLERO, G.A. et al. Prepartum intake, postpartum induction of ketosis, and periparturient disorders affect the metabolic status of dairy cows. Journal of Dairy Science, v.88, n.11, p.3249-3264, 2005.

DRACKLEY, J.K. Transition cow management and periparturient metabolic disorders. In: KASKE, M.; SCHOLZ, H.; HOLTERSHINKEN, M. (Eds.) Recent developments and perspectives in bovine medicine. Tierarztliche Hochschule: Klinik fur Rinderkrankheinten, 2002. p.224-235.

FALLOW, R.J.; HARET, F.J.; DEANE, M.G. Methods of artificially feeding colostrums to the new-born calf Irish Journal of Agriculture Research, v.28, n.11, p.57-63, 1989.

GOREWIT, R.C.; WACHS, E.A.; SAGI, R. et al. Current concepts on the role of oxytocin in milk ejection. Journal of Dairy Science, v.66, n.10, p.2236-2250, 1983.

HITTS, D. Peripartal endocrine changes and the initiation of lactation in ewes of diverse breeds. Growth, v.49, n.2, p.176-189, 1985.

HUDSON, S.; MULLFORD, M.; WHITTESTONE, W. et al. Bovine plasma corticoids during parturition. Journal of Dairy Science, v.59, n.4, p.744-746, 1975.

HYDBRING, E.; MADEJ, A.; MACDONALD, E. et al. Hormonal changes during parturition in heifers and goats are related to the phases and severity of labour. Journal of Endocrinology, v.160, n.1, p.75-85, 1999.
LEFCOURT, A.M.; ELSASSER, T.H. Adrenal responses of Angus x Hereford cattle to the stress of weaning. Journal of Animal Science, v.73, n.9, p.2669-2675, 1995.

LIDFORDS, L.M. Behavioural effects of separating the dairy calf immediately or 4 days post-partum. Applied Animal Behaviour Science, v.49, p.269-283, 1996.

MACUHOVÁ, J.; TANCIN, V.; KRAETZL, W.D. et al. Inhibition of oxytocin release during repeated milking in unfamiliar surroundings: the importance of opioids and adrenal cortex sensitivity. Journal of Dairy Research, v.69, n.1, p.63-73, 2002.

MARNET, P.G.; NEGRÃO, J.A.; LABUSSIÈRE, J. Oxytocin release and milk ejection parameters during milking of dairy ewes in and out of natural season of lactation. Small Ruminants Research, v.28, n.2, p.183-191, 1998.

MARNET, P.G.; NEGRÃO, J.A. The effect of a mixed-management system on the release of oxytocin, prolactin and cortisol in ewes during suckling and machine milking. Reproduction Nutrition Development, v.40, n.3, p.271-281, 2000.

NEGRÃO, J.A. Réponse endocrine lors de l'adaptation à la traite mecanique chez les brebis laitières. Rennes: Ecole National Superiere Agronomique de Rennes, 1996. 133p. Tese (Doutorado em Fisiologia Animal) - Ecole National Superiere Agronomique de Rennes, 1996.

NEGRÃO, J.A.; MARNET, P.G. Effect of calf suckling on oxytocin, prolactin, growth hormone and milk yield in crossbred Gir $\mathrm{x}$ Holstein cows during milking. Reproduction Nutrition Development, v.42, n.4, p.373-380, 2002

NOCEK, J.E.; BRAUND, D.G.; WARNER, R.G. Influence of neonatal colostrum administration, immunoglobulin and continued feeding of colostrum on calf gain, health, and serum protein. Journal of Dairy Science, v.67, n.2, p.319-333, 1984

NATIONAL RESEARCH COUNCIL - NRC. Nutrient requirements of dairy cattle. 7.ed. Washington, D.C.: National Academy Press, 2001. 381p.

ODENSTEN, M.O.; CHILLIARD, Y.; HOLTENIUS, K. Effects of two different feeding strategies during dry-off on metabolism in high-yielding dairy cows. Journal of Dairy Science, v.88, n.11, p.2072-2082, 2005.

OLIVEIRA, J.B.; PRADO, H. Levantamento pedológico semidetalhado do Estado de São Paulo: quadrícula de São Carlos. Campinas: Instituto Agronômico de Campinas, 1984. 188p.

POIDRON, P.; SIGNORET, J. P. Mécanisms de reconnaissance mutuelle de l'agneau et de sa mere. Deuxièmes Journées de la Recherce Ovine et Caprine, v.1, n.2, p.343-353, 1976.

POIDRON, P.; ORGEUR, P.; LE NEINDRE, P. et al. Influence of the blood concentration of prolactin on the length of the sensitive period for establishing maternal behavior in sheep at parturition. Hormonal Behavior, v.14, n.3, p.173-177, 1980.

SANDOVAL-CASTRO, C.A.; ANDERSON, S.; LEAVER, J.D Influence of milking and restricted suckling regimes on milk production and calf growth in temperate and tropical environments. Animal Science, v.69, n.2, p.287-296, 1999.

STATISCAL ANALYSIS SYSTEM - SAS. SAS/STAT ${ }^{\circledR}$ User's guide. Version 8.12. Cary: 2000.1310p.

TANCIN, V.; HARCEK, L.; BROUCEK, J. et al. Effect of suckling during early lactation and changeover to machine milking on plasma oxytocin and cortisol levels and milking characteristics in Holstein cows. Journal of Dairy Research, v.62, n.2, p.249256, 1995.

ZAVY, M.T.; JUNIEWICZ, P.E.; PHILLIPS, W.A. et al. Effect of initial restraint, weaning and transport stress on baseline and ACTH-stimulated cortisol responses in beef cattle of different genotypes. American Journal of Veterinary Research, v.53, n.4, p.551-557, 1992. 